IJCoL

Italian Journal of Computational Linguistics

7-1, 2| 2021

Special Issue: Computational Dialogue Modelling: The Role of Pragmatics and Common Ground in Interaction

\title{
Introduction to the Special Issue on Computational Dialogue Modelling
}

Francesco Cutugno and Hendrik Buschmeier

\section{OpenEdition \\ Journals}

Electronic version

URL: https://journals.openedition.org/ijcol/934

DOI: $10.4000 /$ ijcol. 934

ISSN: 2499-4553

Publisher

Accademia University Press

Printed version

Number of pages: $7-8$

Electronic reference

Francesco Cutugno and Hendrik Buschmeier, "Introduction to the Special Issue on Computational Dialogue Modelling", IJCoL [Online], 7-1, 2 | 2021, Online since 01 December 2021, connection on 26 December 2021. URL: http://journals.openedition.org/ijcol/934 ; DOl: https://doi.org/10.4000/ijcol.934

\section{(c) (i) (3)}

IJCoL is licensed under a Creative Commons Attribution-NonCommercial-NoDerivatives 4.0 International License 


\section{Introduction to the Special Issue on Computational Dialogue Modelling}

\author{
Francesco Cutugno* \\ Università di Napoli 'Federico II'
}

\author{
Hendrik Buschmeier** \\ Bielefeld University
}

This special issue on 'Computational Dialogue Modelling' discusses recent approaches for modelling pragmatics and common ground in spoken human-human and humanmachine interaction. Natural Language Processing (NLP), given the most recent scientific discoveries in the area of intelligent systems and distributed semantics, is now able to build interactive agents whose performance is getting more powerful from year to year. Simple 'command-based' models and dialogue state tracking methods are now widely available for very constrained tasks and domains and research in NLP is heading towards the design of more complex scenarios that need to take into account the role of pragmatics in dialogue systems as well as of grounding and common ground.

To give an example, investigating the role of pragmatics for dialogue systems enables them to adopt linguistic strategies that make internal states more observable. For example, inconsistencies between a conversational agent's internal representations and perceived contextual evidence can become visible by adopting specific superficial forms. Applying computational models of pragmatics to dialogue system design requires more complex approaches to knowledge representation and inference modelling, which can then enable conversational agents to use context-specific clarification strategies, allowing systems to be more robust as inconsistencies can be addressed efficiently. For the future, the field aims at surpassing the basic, task-oriented, applications in order to deal with more complex dialogue situations using more general structures. Managing conflicts in the internal representations among agents involved in the interaction both human and artificial - is of critical importance for a more natural, acceptable user experience when interacting with technical systems with natural language-based interfaces.

This is a special double issue which collects ten contributions. It opens with an invited contribution by Kopp and Varonina, who provide a survey on the main theme of the issue: theories and models of Common Ground establishment in dialogue systems. The article is accompanied by a light debate starting from a number of questions raised by Cutugno and Di Maro, followed by a reply of the authors. The remaining nine articles are organised in two sections: 'Corpus Studies' and 'Technological Models'.

The five articles in the Corpus Studies section deal with the collection, description, or analysis of various corpora, ranging from multimodal interactions to sentiment analysis, from morality to proactivity, and grounding. Although some aspects of pragmatics have already received more attention than others, all the phenomena investigated are essential for future applications showing human-like communicative competence. For

\footnotetext{
* URBAN/ECO Research Center, University of Naples 'Federico II', Naples, Italy, E-mail: cutugno@unina.it

** Faculty of Linguistics and Literary Studies, Bielefeld University, Bielefeld, Germany,

E-mail: hbuschmeduni-bielefeld.de
} 
such systems, large data collections are still needed - especially for languages like Italian.

The four articles in the section Technological Models cover topics related to the implementation of dialogue systems based on innovative models and concepts. Topics range from the general methodology to follow when designing dialogue systems, which still is - in practice - often very task-specific, to the development of specific characteristics, like conflict detection and expression, knowledge representation, and language generation, with a specific interest on the role of common ground.

The scenarios depicted in this issue show the rising interest in new aspects in the design of dialogue systems. The NLP community is creating (for what is new) and reinforcing (for what was created in the past years) the raison d'être of computational pragmatics, blinking an eye, simultaneously, to new forms of knowledge representation, of artificial reasoning, and introducing new components in the design of dialogue state trackers. This research area is in continuous evolution. It interacts with new discoveries in the technical fields of machine learning, knowledge representation, and NLP in general, as well as in dialogue and interaction studies, linguistics and humanagent/robot/computer interaction. It is thus to be expected that, already in the near future, new interventions will be necessary and new threads will be opened.

Finally, we - the two guest editors of this special issue - want to thank Maria Di Maro and Antonio Origlia, the co-editors and, in many cases, deus ex machina of the whole operation. We also want to thank the authors for their contributions, the editors in chief and copy editors of IJCoL, the committee members of the Italian Association of Computational Linguistics, as well as the reviewers - all of whom made this special issue possible. 